Artículo

\title{
Abundancia estacional de Diaphorina citri asociada a la fenología del cultivo de cítricos
}

\author{
Ricardo Álvarez-Ramos ${ }^{1}$ \\ Ausencio Azuara-Domínguez ${ }^{1}$ \\ Jorge Homero Rodríguez-Castro ${ }^{1}$ \\ Vidal Zavala-Zapata ${ }^{1}$ \\ Maricarmen Sánchez-Borja ${ }^{2}$ \\ Jesús Armando Vargas-Tovar ${ }^{1 \S}$ \\ ${ }^{1}$ Tecnológico Nacional de México-IT de Cd. Victoria. Boulevard Emilio Portes Gil núm. 1301, Poniente, \\ Ciudad Victoria, Tamaulipas, México. CP. 87017. Tel. 834 1532000, ext. 180. (rialra@yahoo.com; \\ azuarad@gmail.com; rodriguezjh@ hotmail.com; vidal.convergens@ gmail.com). ${ }^{2}$ Insectos Benéficos del \\ Norte. Carretera Interejidal, Camino Ejidal Libertad s/n, Ciudad Victoria, Tamaulipas. CP. 87260. Tel. 834 \\ 2755452. (sanborjam@gmail.com).
}

${ }^{\S}$ Autor para correspondencia: armandoobiol@gmail.com.

\section{Resumen}

En la presente investigación se determinó la participación de la fenología del cultivo de naranja valencia (Citrus sinensis L. Osbeck) y limón italiano (Citrus limon L. Burn f.) en la abundancia estacional de Diaphorina citri Kuwayama. El estudio se realizó durante los años 2015 al 2018 en tres áreas regionales de control (ARCO'S), ubicadas en los municipios de Padilla, El Barretal, El Carmen, Estación Santa Engracia, Güémez y Victoria, Tamaulipas. En estas áreas se registró la abundancia de $D$. citri en cada fase fenológica del cultivo de naranja (ARCO 1 y 2) y limón (ARCO 3). El cultivo de limón italiano produjo brotes todo el año. Mientras que, el cultivo de naranja valencia presentó una fase de dormancia. En los cuatro años la abundancia estacional de $D$. citri se comportó de manera diferente en los tres ARCO'S. La mayor abundancia del insecto se presentó en la fase de producción de brotación en los árboles. El resultado obtenido en el presente trabajo podría servir para definir el periodo de aplicación de los insecticidas recomendados para el control de $D$. citri en los ARCOS.

Palabras clave: Diaphorina citri, arco, brotes, HLB.

Recibido: noviembre de 2021

Aceptado: febrero de 2022 


\section{Introducción}

En México, el primer reporte del HLB o Huanglongbing ocurrió en julio de 2009 y para noviembre de 2015 la enfermedad se registró en 347 municipios de 18 estados productores de cítricos en México (Garza et al., 2017). El impacto económico y social estimado por la presencia de esta enfermedad, en tan solo tres años de su llegada, fue de 1.7 millones de toneladas de frutos y 112.1 millones de jornales perdidos (Díaz et al., 2014). Para mitigar lo anterior, se diseñó e implementó la campaña fitosanitaria contra el HLB. Las actividades realizadas en la campaña contra el HLB al inicio estuvieron enfocada a la detección y búsqueda de síntomas que ocasiona el agente causal de la enfermedad, la bacteria Candidatus Liberibacter asiaticus Jagoueix (Rhizobiales: Rhizobiaceae).

Posteriormente, se enfocaron al monitoreo del insecto vector, Diaphorina citri, de la bacteria con el fin de conocer la distribución poblacional del insecto plaga en tiempo y espacio (Flores et al., 2017), y medir el impacto de los insecticidas contemplados en la estrategia contra el HLB en las poblaciones regionales del insecto. En Tamaulipas, D. citri fue registrado en 2003 (López-Arroyo, 2005). En 2014, el Comité Estatal de Sanidad Vegetal de Tamaulipas (CESAVETAM) estableció un programa de monitoreo con trampas amarillas pegajosas bajo el esquema de áreas regionales de control (ARCO'S) con el fin de controlar la dispersión del insecto plaga y de la enfermedad bacteriana en 33272.12 ha de naranja (Citrus sinensis L. Osbeck) (Sapindales: Rutaceae) y 3 339.31 ha de limón (Citrus limon L. Burn f.) (Sapindales: Rutaceae) (SIAP, 2019), ubicadas en Padilla, El Barretal, El Carmen, Estación Santa Engracia, Güémez y Victoria (Mora et al., 2014).

Por la importancia económica de la producción de cítricos en estos municipios, es necesario analizar la abundancia estacional de $D$. citri en árboles de naranja valencia y limón italiano con el fin de evitar la presencia del HLB. Por lo tanto, el objetivo de esta investigación fue determinar la abundancia estacional de $D$. citri asociada a la fenología de la naranja valencia y limón italiano.

\section{Materiales y métodos}

\section{Área de estudio}

El experimento se estableció durante 2015 al 2018 en tres áreas regionales de control (ARCO'S) ubicadas en el municipio de Padilla, El Barretal, El Carmen, Estación Santa Engracia, Güémez y Victoria, Tamaulipas (Figura 1).

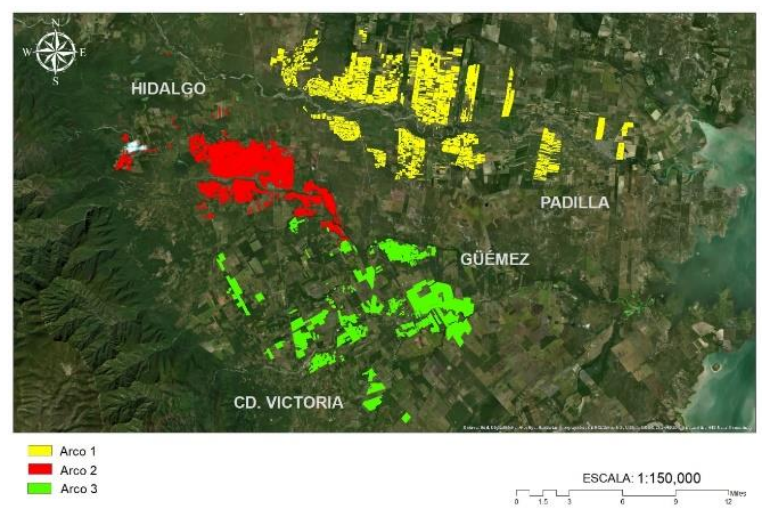

Figura 1. Ubicación geográfica de las áreas regionales de control (ARCO’S) en Tamaulipas, México. 
El ARCO 1 estuvo conformado por 5402.38 ha, 65 huertas comerciales (Cuadro 1) sembradas con árboles de naranja valencia. La edad promedio de los árboles fue de 15 años, la separación entre los árboles y líneas fue de cuatro y siete metros. Los árboles fueron regados con un sistema de riego rodado.

Cuadro 1. Superficie y localización geográfica de las huertas de naranja valencia Citrus sinensis (L.) Osbeck del ARCO 1 ubicadas en el municipio de Padilla y EI Barretal.

\begin{tabular}{|c|c|c|c|}
\hline Huerta comercial & Superficie (ha) & Latitud & Longitud \\
\hline Ejido La Soledad 6 & 57 & 24.086535 & -99.057106 \\
\hline Ejido Mártir de Chinameca 2 & 125.75 & 24.113646 & -99.09361 \\
\hline Ejido Conrado Castillo 4 & 14.5 & 24.08759 & -99.02356 \\
\hline Ejido José López Portillo 7 & 4 & 24.04135 & -99.00219 \\
\hline Ejido La Soledad 2 & 57 & 24.118633 & -99.070244 \\
\hline Ejido La Soledad 9 & 64 & 24.104939 & -99.054146 \\
\hline Ejido San Patricio 2 & 132 & 24.060936 & -99.079445 \\
\hline Huerta El Tejón 2 & 50 & 24.03793 & -98.886696 \\
\hline Ejido Conrado Castillo 3 & 14.5 & 24.070562 & -99.03035 \\
\hline Ejido José López Portillo 6 & 34 & 24.048689 & -99.0219 \\
\hline Ejido La Soledad 4 & 57 & 24.132109 & -99.067894 \\
\hline Ejido Conrado Castillo 10 & 15.39 & 24.056007 & -99.03695 \\
\hline Ejido Conrado Castillo 5 & 14.5 & 24.077688 & -99.02941 \\
\hline Ejido Plan de Ayala 3 & 192.83 & 24.043032 & -99.09813 \\
\hline Ejido Plan de Ayala 1 & 192.84 & 24.032415 & -99.10381 \\
\hline Ejido Guadalupe Victoria 1 & 41 & 24.063482 & -99.12173 \\
\hline Ejido La Soledad 5 & 57 & 24.134998 & -99.06753 \\
\hline Ejido Guadalupe Victoria 5 & 41 & 24.064547 & -99.14512 \\
\hline Ejido San Patricio 3 & 132 & 24.058231 & -99.07481 \\
\hline Ejido La Soledad 7 & 57 & 24.09412 & -99.055435 \\
\hline Ejido Guadalupe Victoria 9 & 41 & 24.072016 & -99.15731 \\
\hline Ejido Conrado Castillo 7 & 14.5 & 24.050945 & -99.03912 \\
\hline Ejido Marte R. Gómez 7 & 72.95 & 24.09832 & -99.03486 \\
\hline Ejido José López Portillo 4 & 34 & 24.041721 & -99.00998 \\
\hline Ejido San Patricio 1 & 132 & 24.042892 & -99.085311 \\
\hline Ejido José López Portillo 5 & 34 & 24.041246 & -99.02064 \\
\hline Ejido Mártir de Chinameca 1 & 125.75 & 24.104736 & -99.11817 \\
\hline Ejido Marte R. Gómez 6 & 72.95 & 24.08675 & -99.0412 \\
\hline Ejido Guadalupe Victoria 8 & 41 & 24.065233 & -99.145355 \\
\hline Ejido La Soledad 8 & 57 & 24.08344 & -99.0645 \\
\hline Ejido La Concepción 2 & 83 & 24.106049 & -99.05002 \\
\hline
\end{tabular}




\begin{tabular}{|c|c|c|c|}
\hline Huerta comercial & Superficie (ha) & Latitud & Longitud \\
\hline Huerta El Paraíso & 100 & 24.08466 & -99.08274 \\
\hline Ejido Marte R. Gómez 5 & 72.95 & 24.082705 & -99.04501 \\
\hline Ejido Marte R. Gómez 1 & 5 & 24.072016 & -99.044785 \\
\hline Ejido La Concepción 1 & 83 & 24.09197 & -99.04489 \\
\hline Ejido La Concepción 4 & 83 & 24.082035 & -99.05381 \\
\hline Ejido Guadalupe Victoria 2 & 41 & 24.065823 & -99.12232 \\
\hline Ejido Cruz y Cruz 1 & 382 & 24.09858 & -99.18552 \\
\hline Ejido José López Portillo 2 & 34 & 24.0361 & -99.0126 \\
\hline Ejido Conrado Castillo 8 & 14.5 & 24.050976 & -99.01905 \\
\hline Ejido Guadalupe Victoria 3 & 41 & 24.067486 & -99.131035 \\
\hline Ejido Guadalupe Victoria 6 & 41 & 24.054068 & -99.13169 \\
\hline Ejido Guadalupe Victoria 4 & 41 & 24.06885 & -99.13532 \\
\hline Ejido José López Portillo 1 & 34.13 & 24.035378 & -99.01616 \\
\hline Ejido La Concepción 5 & 83 & 24.076841 & -99.05645 \\
\hline Huerta Lote 47 & 45 & 24.057177 & -98.88358 \\
\hline Ejido Guadalupe Victoria 10 & 41 & 24.070724 & -99.15082 \\
\hline Ejido Nuevo San Juan 1 & 258 & 24.047245 & -99.04309 \\
\hline Ejido Guadalupe Victoria 11 & 41.96 & 24.077404 & -99.16936 \\
\hline Ejido Plan de Ayala 2 & 192.83 & 24.041084 & -99.105446 \\
\hline Ejido La Soledad 3 & 57 & 24.127895 & -99.07232 \\
\hline Ejido Marte R. Gómez 3 & 72.95 & 24.068739 & -99.04935 \\
\hline Ejido Conrado Castillo 6 & 14.5 & 24.051632 & -99.04219 \\
\hline Ejido Carmen Galindeño 1 & 681.7 & 24.094416 & -99.141716 \\
\hline Ejido Conrado Castillo 1 & 14.5 & 24.075184 & -99.02093 \\
\hline Ejido Conrado Castillo 9 & 14.5 & 24.056099 & -99.02827 \\
\hline Huerta El Tejón 1 & 50 & 24.038193 & -98.889534 \\
\hline Ejido Marte R. Gómez 2 & 72.95 & 24.06817 & -99.0434 \\
\hline Ejido Marte R. Gómez 4 & 72.95 & 24.076044 & -99.05218 \\
\hline Ejido Guadalupe Victoria 7 & 41 & 24.056377 & -99.13623 \\
\hline Huerta El Lucero & 100 & 24.096743 & -98.98633 \\
\hline Ejido José López Portillo 3 & 34 & 24.041996 & -99.01711 \\
\hline Ejido José Silva Sánchez 2 & 233 & 24.124647 & -99.05361 \\
\hline Ejido José Silva Sánchez 1 & 233 & 24.08227 & -99.07351 \\
\hline Ejido Conrado Castillo 2 & 14.5 & 24.06979 & -99.02477 \\
\hline
\end{tabular}


El ARCO 2 estuvo conformado por 3491.23 ha, 49 huertas comerciales (Cuadro 2) sembradas con naranja valencia. La edad promedio de los árboles fue de 15 años, la separación entre los árboles y líneas fue de cuatro y siete metros. Los árboles fueron regados con un sistema de riego rodado.

Cuadro 2. Superficie y localización geográfica de las huertas de naranja valencia Citrus sinensis (L.) Osbeck del ARCO 2 ubicadas en el El Carmen y Estación Santa Engracia.

\begin{tabular}{|c|c|c|c|}
\hline Huerta comercial & Superficie (ha) & Latitud & Longitud \\
\hline Ejido El Arco 8 & 22.65 & 23.99976 & -99.25969 \\
\hline Ejido El Arco 1 & 22.65 & 24.009975 & -99.256165 \\
\hline Ejido El Arco 4 & 22.65 & 24.011806 & -99.26059 \\
\hline Ejido San Isidro 2 & 43 & 24.015835 & -99.24684 \\
\hline Ejido El Arco 6 & 22.65 & 24.018017 & -99.26326 \\
\hline Ejido El Arco 3 & 22.65 & 23.988935 & -99.171196 \\
\hline Ejido El Porvenir 3 & 18.5 & 23.99402 & -99.259415 \\
\hline Ejido El Porvenir 2 & 18 & 23.984842 & -99.17778 \\
\hline Ejido El Arco 10 & 22.65 & 23.991343 & -99.254845 \\
\hline Ejido San Isidro 1 & 43.28 & 24.008848 & -99.24169 \\
\hline Ejido El Arco 9 & 22.65 & 24.020386 & -99.25239 \\
\hline Ejido El Arco 7 & 22.65 & 24.008661 & -99.248955 \\
\hline Ejido El Arco 2 & 22.65 & 24.012655 & -99.24023 \\
\hline Ejido Guillermo Zúñiga 6 & 74.14 & 24.044983 & -99.20357 \\
\hline Ejido El Arco 5 & 22.65 & 24.018524 & -99.25535 \\
\hline Ejido Guillermo Zúñiga 3 & 74.14 & 24.019312 & -99.19423 \\
\hline Ejido Vicente Guerrero 1 & 61 & 24.035128 & -99.21106 \\
\hline Ejido Emiliano Zapata 3 & 90.51 & 24.035948 & -99.23369 \\
\hline Ejido Guillermo Zúñiga 7 & 74.14 & 24.033747 & -99.18054 \\
\hline Ejido Emiliano Zapata 1 & 90.51 & 24.037302 & -99.22089 \\
\hline Ejido Emiliano Zapata 4 & 90.51 & 24.048454 & -99.19514 \\
\hline Ejido Benito Juárez 3 & 94 & 24.04681 & -99.220184 \\
\hline Ejido Guillermo Zúñiga 8 & 74.14 & 24.02302 & -99.24403 \\
\hline Ejido Balconcitos 4 & 27.92 & 24.016384 & -99.216675 \\
\hline Ejido San José de Santa Engracia 1 & 728.11 & 24.024101 & -99.236465 \\
\hline Ejido Vicente Guerrero 2 & 61 & 24.044575 & -99.211586 \\
\hline Ejido El Porvenir 1 & 18.5 & 23.984312 & -99.181885 \\
\hline Ejido Vicente Guerrero 3 & 61 & 24.041195 & -99.21406 \\
\hline Ejido La Diana 1 & 374.42 & 23.995256 & -99.14137 \\
\hline Ejido Guillermo Zúñiga 5 & 74.14 & 24.033224 & -99.19679 \\
\hline Ejido Benito Juárez 1 & 94 & 24.049835 & -99.23015 \\
\hline Ejido Guillermo Zúñiga 9 & 74.14 & 24.050026 & -99.20151 \\
\hline Ejido Guillermo Zúñiga 1 & 85.99 & 24.017195 & -99.1816 \\
\hline Ejido Guillermo Zúñiga 2 & 74.18 & 24.025429 & -99.1884 \\
\hline Ejido Balconcitos 6 & 27.92 & 24.018547 & -99.2217 \\
\hline
\end{tabular}




\begin{tabular}{cccc}
\hline Huerta comercial & Superficie (ha) & Latitud & Longitud \\
\hline Ejido Balconcitos 3 & 27.92 & 24.015446 & -99.229996 \\
Huerta las Enramadas & 50 & 24.023846 & -99.21707 \\
Ejido Benito Juárez 2 & 94 & 24.042488 & -99.244865 \\
Ejido La Rosita 1 & 43.07 & 24.0049 & -99.2263 \\
Ejido La Rosita 3 & 43.07 & 24.008351 & -99.22203 \\
Ejido Balconcitos 1 & 27.92 & 24.011097 & -99.20382 \\
Ejido Guillermo Zúñiga 4 & 74.14 & 24.027617 & -99.198105 \\
Ejido Balconcitos 5 & 27.92 & 24.016468 & -99.23209 \\
Ejido Balconcitos 2 & 27.92 & 24.013247 & -99.21983 \\
Ejido Emiliano Zapata 2 & 90.51 & 24.042408 & -99.2314 \\
Ejido La Rosita 2 & 43.07 & 24.004097 & -99.232506 \\
Ejido Ceylán 1 & 34 & 23.97847 & -99.14093 \\
Ejido Acatlán & 100 & 23.96543 & -99.13133 \\
Ejido Ceylán 2 & 34 & 23.9817 & -99.14491 \\
\hline
\end{tabular}

El ARCO 3 estuvo conformado por 1575.75 ha, 18 huertas comerciales (Cuadro 3) sembradas con árboles de limón italiano. La edad promedio de los árboles fue de cinco años, la separación entre los árboles y líneas fue de cuatro y siete metros. Los árboles fueron regados con un sistema de riego por microaspersión.

Cuadro 3. Superficie y localización geográfica de las huertas de limón italiano Citrus limon (L.) Burn f. del ARCO 3 ubicadas en el municipio de Güiémez y Ciudad Victoria.

\begin{tabular}{cccc}
\hline Huerta comercial & Superficie (ha) & Latitud & Longitud \\
\hline Huerta La Viuda & 55 & 23.915712 & -99.09632 \\
Huerta La Providencia 3 & 50 & 23.914999 & -99.07397 \\
Huerta Tres Sabinos & 30 & 23.900478 & -99.05132 \\
Huerta Macabeos 4 & 56 & 23.925621 & -99.02932 \\
Ejido Esfuerzo Del Campesino & 2 & 23.937151 & -99.12753 \\
Huerta El Filosofo & 53.25 & 23.916235 & -99.06931 \\
Huerta Najita & 116 & 23.922585 & -99.046234 \\
Huerta La Ilusión & 127.4 & 23.928032 & -99.05204 \\
Huerta La Querencia & 60 & 23.917574 & -99.050766 \\
Ejido La San Juana 3 & 3.4 & 23.924044 & -99.133865 \\
Huerta El Contador & 21 & 23.929514 & -99.24125 \\
Huerta El Cuatro & 19 & 23.936605 & -99.23088 \\
Huerta El Jericob & 74.61 & 23.885063 & -99.13875 \\
Huerta La Pomarosa & 40 & 23.889816 & -99.04195 \\
Huerta Nuevo Guadalupe & 181 & 23.884377 & -99.031525 \\
Huerta El 12 & 101.45 & 23.821293 & -99.0759 \\
Huerta Teresitas & 251 & 23.899931 & -99.183525 \\
Huerta Laborcitas & 334.64 & 23.851086 & -99.09927 \\
\hline
\end{tabular}




\section{Registro de la fenología de los árboles de cítricos}

El registro de la fenología de los cultivos de naranja valencia y limón italiano se realizó cada ocho días tomando como referencia lo publicado por Robles y Delgadillo (2010); Lozano y Jasso (2012).

\section{Monitoreo de adultos de $D$. citri en los ARCO'S}

Los adultos fueron capturados con trampas de color amarillo de $7.5 \mathrm{~cm}$ por $12.5 \mathrm{~cm}$, estampadas con una cuadrícula negra de $1.5 \mathrm{~cm}$ y acompañadas de una base cubierta con el adherente Stick Bug 50\% ${ }^{\circledR}$. En las huertas, las trampas fueron colocadas con base en el manual operativo de la campaña del HLB. Se colocaron 10 trampas en la periferia (sur-este) y 10 trampas en línea transversal a esta, a una distancia entre trampas de $12 \mathrm{~m}$ y $1.2 \mathrm{~m}$ del nivel del suelo. Las trampas fueron remplazadas cada ocho días y el número de adultos fue cuantificado en el laboratorio. En el ARCO 1, ARCO 2 y ARCO 3 se instalaron 1 300, 980 y 360 trampas.

\section{Análisis estadístico}

El número de individuos de D. citri fueron agrupados en seis categorías con rangos de 0 a 100 individuos tomando como referencia que el número menor de adultos capturados por mes es de 20 y el mayor de 134 (Flores et al., 2017). Las categorías de las densidades poblacionales del insecto fueron: plaga 1 (0 individuos), plaga 2 (1 a 100 individuos), plaga 3 (100 a 200 individuos), plaga 4 (200 a 300 individuos), plaga 5 (300 a 400 individuos) y la plaga 6 (400 a 500 individuos). Las categorías fueron asociadas con las fases de brotación, brotación-fructificación, brotaciónfloración, brotación-desarrollo y dormancia de huertos comerciales de naranja valencia (ARCO 1 y 2) y limón italiano (ARCO 3).

Finalmente, las categorías fueron sometidas a un análisis multivariado de correspondencia múltiple (Benzécri, 1973; Greenacre, 1983). El análisis considera el porcentaje de inercia de los ejes X y Y interpretándose el que presenta el mayor porcentaje de inercia. La interpretación de los resultados consiste en asociar las categorías con mayor cercanía considerando datos de frecuencia sin perder la identidad u origen de los sitios muestreados (Rencher, 2002).

\section{Resultados y discusión}

\section{Fenología del cultivo de naranja valencia y limón italiano}

Los árboles de naranja valencia presentaron la siguiente fenología: producción de brotes, floración, desarrollo del fruto, dormancia y fructificación. La producción de brotes se presentó de enero a julio y de octubre a diciembre, la fructificación ocurrió en enero y en diciembre. La floración inicio a finales de marzo y finalizó en la tercera semana de abril, la fase de desarrollo inició a finales de abril y finalizó en julio y la fase de dormancia ocurrió a finales de julio y finalizó en la primera semana de octubre. Por otro lado, los árboles de limón italiano presentaron la siguiente fenología: producción de brotes, floración, desarrollo del fruto y fructificación. La producción de brotes ocurrió todo el año, la fructificación se presentó en enero y en diciembre, la floración inició a finales de marzo y finalizó en la tercera semana de abril y la fase de desarrollo inició a finales de abril y finalizó en julio. 


\section{Abundancia estacional de $D$. citri asociada a las fases fenológicas de los cítricos en los ARCO'S}

En 2015, la población de $D$. citri presentó dos asociaciones significativas con las fases fenológicas en los tres ARCO'S $X^{2}=1664.78, \mathrm{df}=121$ (Figura 2). Poblaciones de 1 a 100 individuos se asociaron con la fase de brotación-desarrollo en el ARCO 1 (A1). Mientras que, poblaciones de 1 a 100 individuos se asociaron con la fase de brotación-desarrollo, brotación-floración y dormancia en el ARCO 2 (A2) y las densidades poblacionales de 100 a 200 individuos se asociaron con la fase de brotación en el ARCO 3 (A3).

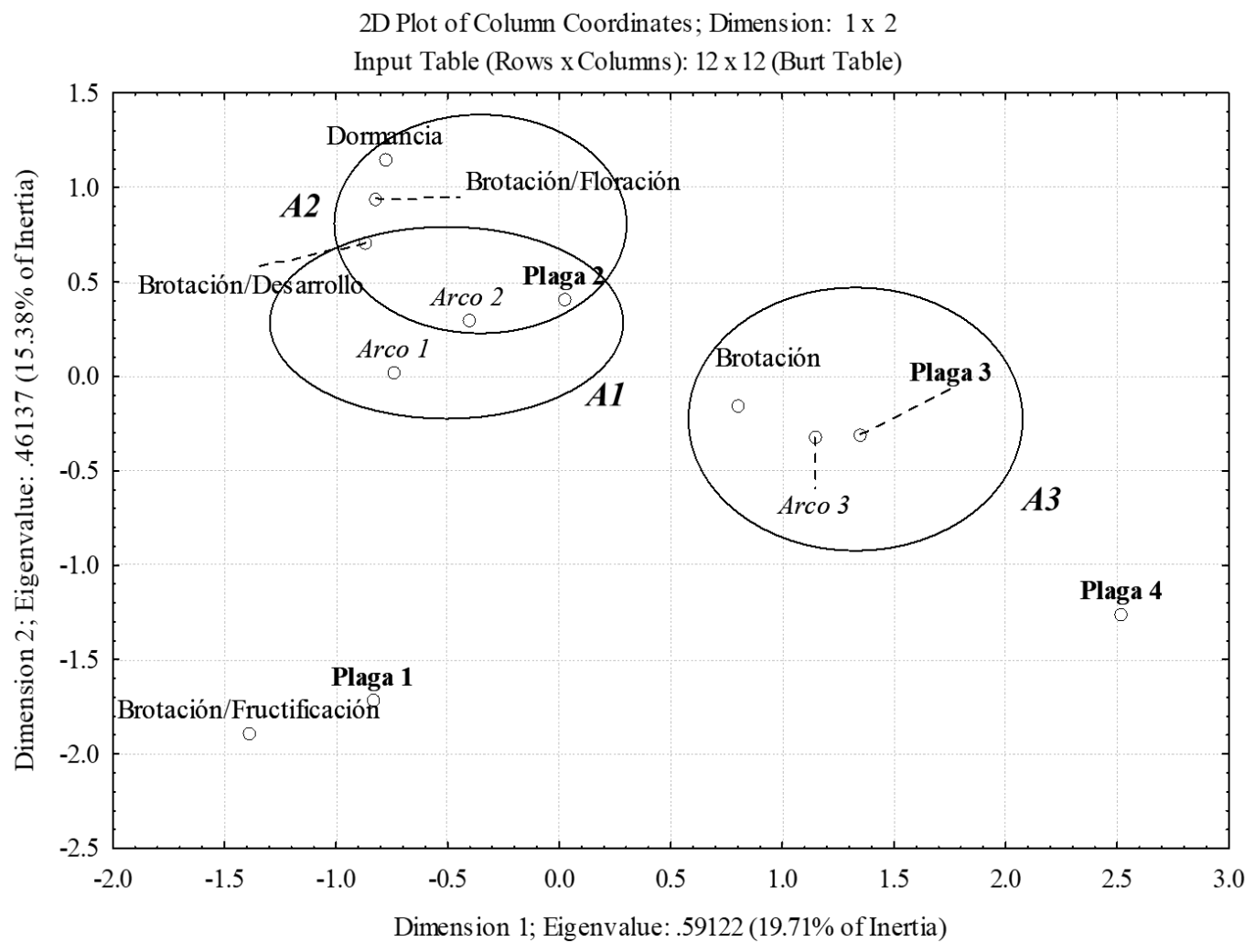

Figura 2. Análisis de la asociación de la abundancia estacional de Diaphorina citri en las fases fenológicas del cultivo de naranja valencia y limón italiano en tres áreas regionales de control en 2015. Las categorías de las densidades poblacionales del insecto fueron plaga 1 ( 0 individuos), plaga 2 (1 a 100 individuos), plaga 3 (100 a 200 individuos), plaga 4 (200 a 300 individuos), plaga 5 (300 a 400 individuos) y la plaga 6 (400 a 500 individuos).

En 2016, los adultos de $D$. citri presentaron asociación significativa con las fases fenológicas en los tres ARCO'S X2=1998.24, df= 169 (Figura 3). Poblacionales de 100 a 200 individuos se asociaron con la fase de brotación-desarrollo en el ARCO 1 (A1). Mientras que, poblaciones de 400 a 500 individuos se asociaron con la fase brotación-desarrollo en el ARCO 2 (A2). Del mismo modo, densidades de 400 a 500 individuos se asociaron con la fase de brotación en el ARCO 3 (A3). 


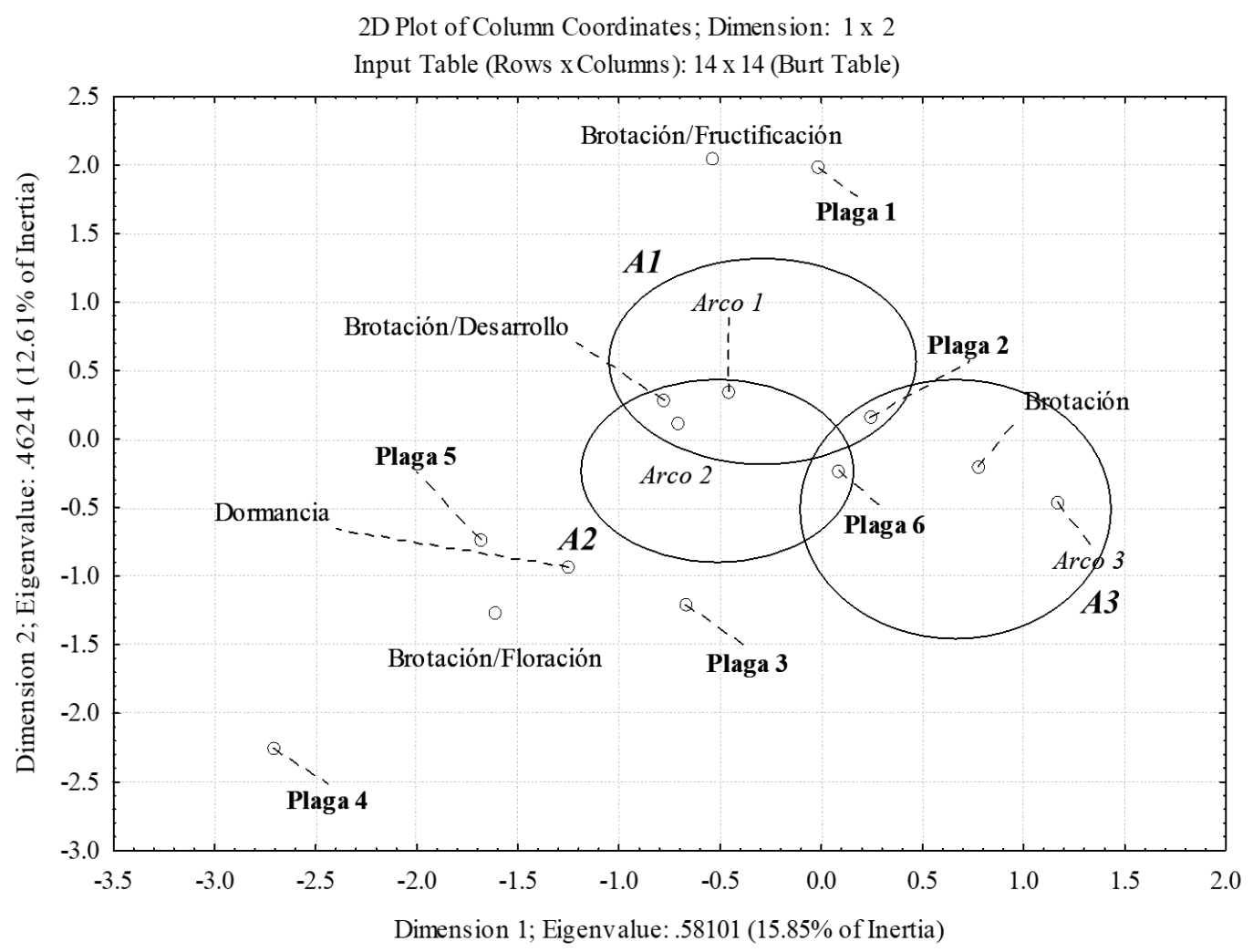

Figura 3. Análisis de la asociación de la abundancia estacional de Diaphorina citri en las fases fenológicas del cultivo de naranja valencia y limón italiano en tres áreas regionales de control en 2016. Las categorías de las densidades poblacionales del insecto fueron plaga $1(0$ individuos), plaga 2 ( 1 a 100 individuos), plaga 3 (100 a 200 individuos), plaga 4 (200 a 300 individuos), plaga 5 (300 a 400 individuos) y la plaga 6 (400 a 500 individuos).

En 2017, se presentaron dos asociaciones entre el número de individuos de D. citri con las fases fenológicas en los tres ARCO'S (X2=2087.39, df=169 (Figura 4). La primera asociación se llevó a cabo con densidades poblacionales de 1 a 200 individuos en la fase de brotación en el ARCO 3 (A3). Para la segunda asociación, poblaciones de 200 a 300 individuos se asociaron con las fases de brotación-floración y brotación-desarrollo en los ARCO’S 1 y 2 (A1, A2).

En 2018, la población de $D$. citri presentó asociaciones significativas con las fases fenológicas en los tres ARCO'S $\left(\mathrm{X}^{2}=2313.21, \mathrm{df}=196\right.$ (Figura 5). Densidades poblacionales de 1 a 100 individuos se asociaron con las fases de brotación, brotación-floración, brotación-fructificación y dormancia en el ARCO 1 (A1). Densidades poblacionales de 100 a 200 individuos se asociaron con la fase brotación-desarrollo en el ARCO 2 (A2). Por último, densidades de 200 a 300 individuos fueron asociadas con la fase de brotación en el ARCO 3 (A3).

La abundancia de $D$. citri fue diferente en los ARCO'S y en los años de estudio. De acuerdo con Razeto (2005), la variedad del cultivar y la producción de brotes en los árboles de cítricos influye en la abundancia de $D$. citri. En el presente trabajo, la diferencia en la abundancia de $D$. citri posiblemente fue debido a la cantidad y al período de producción de brotes en el cultivo de naranja valencia y limón italiano, ya que el cultivo de naranja produce menos brotes (38.70 brotes/mes) al año que el cultivo de limón (673.5 brotes/mes) (Medina et al., 2007; Chávez et al., 2016). 


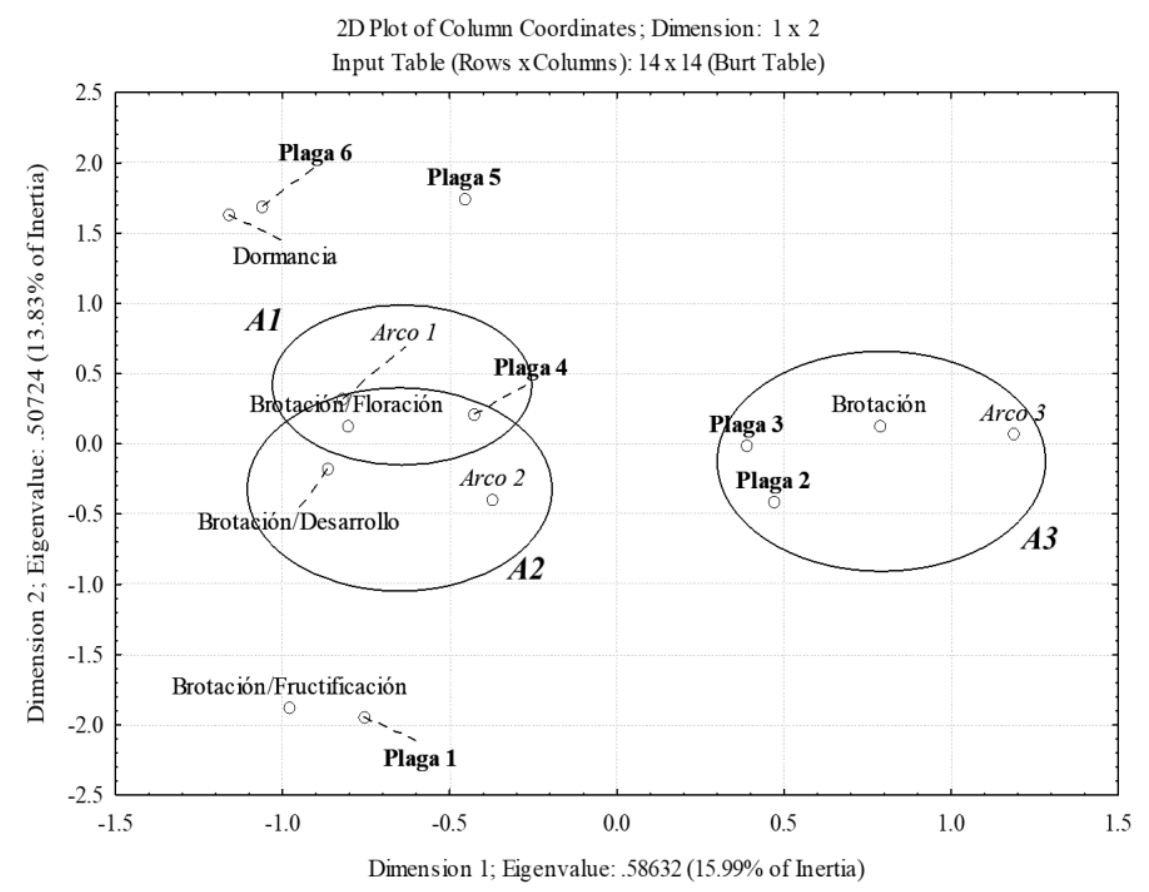

Figura 4. Análisis de la asociación de la abundancia estacional de Diaphorina citri en las fases fenológicas del cultivo de naranja valencia y limón italiano en tres áreas regionales de control en 2017. Las categorías de las densidades poblacionales del insecto fueron plaga 1 ( 0 individuos), plaga 2 (1 a 100 individuos), plaga 3 (100 a 200 individuos), plaga 4 (200 a 300 individuos), plaga 5 (300 a 400 individuos) y la plaga 6 (400 a 500 individuos).

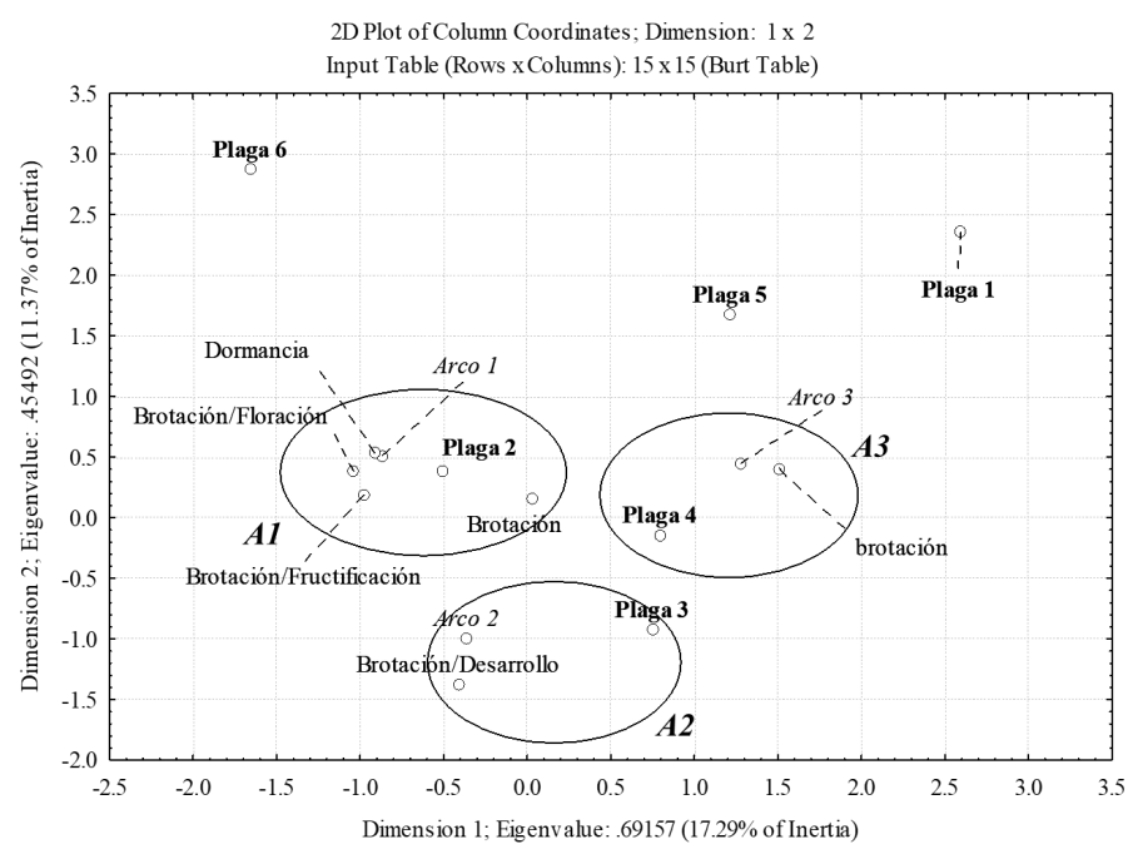

Figura 5. Análisis de la asociación de la abundancia estacional de Diaphorina citri en las fases fenológicas del cultivo de naranja valencia y limón italiano en tres áreas regionales de control en 2018. Las categorías de las densidades poblacionales del insecto fueron plaga 1 ( 0 individuos), plaga 2 (1 a 100 individuos), plaga 3 (100 a 200 individuos), plaga 4 (200 a 300 individuos), plaga 5 (300 a 400 individuos) y la plaga 6 (400 a 500 individuos). 
En los ARCOS, los árboles del cultivo de naranja valencia produjeron brotes de enero a julio y de octubre a diciembre. Mientras que, los árboles del cultivo de limón italiano presentaron brotes todo el año, posiblemente por el sistema de riego por microaspersión, uso de fertilizantes, poda de los árboles y porque los árboles de limón son más jóvenes (cinco años) que los árboles de naranja (Pluke et al., 2008). Mientras que, las huertas del cultivo de naranja no cuentan con un sistema de riego, el uso de fertilizantes es limitado y los árboles tienen en promedio 15 años.

En los tres años, la mayor abundancia de $D$. citri registrada en el ARCO 3 estuvo asociada a la fase de brotación del cultivo de limón italiano. Mientras que, la mayor abundancia registrada en el ARCO 1 y ARCO 2 estuvo asociada a la fase de brotación y brotación-desarrollo del cultivo de naranja valencia. En ambos cultivos, $D$. citri ocurre en la fase de producción y desarrollo de brotes, debido a que los adultos y las ninfas se alimentan de estos para madurar sus huevos y completar el desarrollo (Ortega et al., 2013). Aunque también, los adultos se alimentan y sobreviven por varios meses en las hojas desarrolladas (Tsai et al., 2002; Fernández y Miranda, 2005; Stansly y Qureshi, 2007; Qureshi y Stansly, 2008). Hall et al. (2008) indicaron que los picos poblacionales de D. citri ocurren cuando existen condiciones ambientales favorables y disponibilidad de brotes.

Al respecto, el cultivo de limón italiano produjo brotes todo el año (de diciembre a marzo se observó la abundancia mayor de brotes) y D. citri estuvo presente en los árboles. De forma similar, esto ha ocurrido en el cultivo de limón mexicano en el municipio de Apatzingán, Michoacán (Luna et al., 2018). D. citri está presente durante todo el año debido a que el cultivo produce brotes de forma constante. Sin embargo, el pico poblacional máximo ocurre en el mes de agosto a causa de las condiciones ambientales favorables para el insecto. Resultado similar fue determinado en el limón persa (C. latifolia) en el municipio de Huimanguillo, Tabasco (García et al., 2013).

Al igual que en las huertas de limón mexicano, este cultivo produce brotes de forma constante y la abundancia mayor de $D$. citri ocurren de marzo a julio. De forma contraria, en el limón mexicano en Acapulco, Guerrero, la población menor de adultos de D. citri se presentó en febrero, cuando los árboles estaban en la etapa de producción de brotes. Mientras que, la abundancia mayor ocurrió en abril y en noviembre, en la etapa de amarre y desarrollo del fruto, respectivamente. En este caso la baja precipitación (Botero et al., 2014) y temperatura óptima jugaron un papel importante en la ocurrencia de picos poblacionales altos de $D$. citri en los árboles (25 a $28^{\circ} \mathrm{C}$ ) (Liu y Tsai, 2000; Paris et al., 2017).

De forma contraria al cultivo de limón italiano, los árboles del cultivo de naranja valencia no produjeron brotes todo el año. Sin embargo, la mayor abundancia de $D$. citri ocurrió en fase de producción de brotes y en el desarrollo de estos. Resultado similar fue reportado por Ortega et al. (2013) en Cazones, Veracruz. Los autores registraron los periodos de mayor abundancia de D. citri en los árboles de naranja ( $C$. sinensis $c v$ Mars, $C$. sinensis $c v$ Valencia) y lima de chiche $(C$. limetta) de febrero a abril y en julio, que coinciden con la fase de producción y desarrollo de brotes.

\section{Conclusiones}

Durante los años 2015 al 2018 el tamaño poblacional de D. citri fue diferente en los tres ARCO'S. En este resultado la fenología del cultivo jugó un papel importante. Debido a que, la abundancia mayor del insecto se registró en la fase de producción de brotes vegetativos. 


\section{Agradecimientos}

Al Comité Estatal de Sanidad Vegetal de Tamaulipas (CESAVETAM) por el apoyo en la realización de la presente investigación y, al Consejo Nacional de Ciencia y Tecnología (CONACYT) por la beca otorgada al C. Ricardo Álvarez Ramos para realizar el Doctorado en Ciencias en Biología en el Instituto Tecnológico de Ciudad Victoria, Tamaulipas.

\section{Literatura citada}

Benzécri, J. P. 1973. L'Analyse des données. l'analyse des correspondances. Ensieta. Paris. 119 p. Botero, V.; Ochoa, A.; Gastón, J. Z.; Ortiz, R. A.; Fuel, T. S. M.; Moná, F. E.; Marcela, M. L.; Guarin, H. J.; Orduz, R. J. O.; Chaparro, Z. H. N. y Arévalo, P. E. 2014. Identificación de la dinámica poblacional de Diaphorina citri (Hemiptera: Liviidae) en los cultivos de cítricos de Colombia: una herramienta para implementar un sistema piloto de seguimiento de poblaciones del insecto vector del HLB. Universidad nacional de Colombia. Medellín, Colombia. 89 p.

Chávez, J. A.; Flores, G. L.; Góngora, A. M.; Gómez, R. L. y García, C. B. 2016. Distribución temporal de Diaphorina citri Kuwayama (Hemíptera: Psyllidae) en limón persa (Citrus latifolia Tanaka) en el municipio de Sinaloa, Sinaloa. Entomol. Mex. 3(1):324-329.

Díaz, G.; López, J. I.; Sánchez, I.; Guajardo, R. A.; Mora, G. y Quijano, J. A. 2014. Áreas de abundancia potencial en México del vector del Huanglongbing, Diaphorina citri (Hemiptera: Liviidae). Rev. Mex. Cienc. Agríc. 5(7):1137-1153.

Fernández, M. y Miranda, I. 2005. Comportamiento de Diaphorina citri Kuwayama (Hemiptera: Psyllidae). Parte III. Relación entre el ciclo de vida y el brote vegetativo foliar. Rev. Protección Vegetal. 20(3):161-164.

Flores, J. C.; Aguilar, C.; Alcántara, J. A.; Ayvar, S. y Catalán, G. 2017. Fluctuación poblacional del psílido Diaphorina citri Kuwayama (Hemiptera: Liviidae) en limón mexicano en Acapulco, Guerrero. Acta Agrícola y Pecuaria. 3(2):58-60.

García, D.; Sánchez, S.; Romero, J. y Pérez, J. 2013. Fluctuación poblacional de Diaphorina citri (Hemiptera: Liviidae) en limón persa (Citrus latifolia), en Huimanguillo, Tabasco, México. Rev. Colomb. Entomol. 39(2):201-204.

Garza, J. J.; Varela, S. y Gómez, W. 2017. Métodos para la detección presuntiva de Huanglongbing (HLB) en cítricos. CienciaUAT. 2(2):93-104.

Greenacre, J. 1983. Theory and applications of correspondence analysis. London. Academic Press. $364 \mathrm{p}$.

Hall, D. G.; Hentz, M. G., and Aldair, R. C. 2008. Population ecology and phenology of Diaphorina citri (Hemiptera: Psyllidae) in two Florida citrus groves. Entomological Society of America. 37(4):914-924.

Liu, Y. H. and Tsai, J. H. 2000. Effects of temperature on biology and life table parameters of the Asian citrus psyllid, Diaphorina citri Kuwayama (Homoptera: Psyllidae). Annals Appl. Biol. 137(3):201-206.

López-Arroyo, J. I.; Peña, M. A.; Rocha-Peña, M. A. y Loera, J. 2005. Ocurrencia en México del psílido asiático Diaphorina citri (Hemiptera: Psyllidae). In: Memorias del VII congreso internacional de fitopatología. Chihuahua, Chih. México. 86 p.

Lozano, M. G. y Jasso, J. 2012. Identificación de enemigos naturales de Diaphorina citri Kuwayama (Hemiptera: Psyllidae) en el estado de Yucatán, México. Fitosanidad. 16(1):5-11. 
Luna, A.; Escamilla, J. L.; Barrera, S. I. y Loera, E. 2018. Fluctuación poblacional de Diaphorina citri Kuwayama (Hemiptera: Liviidae) en el Valle de Apatzingán, Michoacán. Acta Zoológica Mexicana (nueva serie). 34(1):1-4.

Medina, V. M.; Zapiaín, G.; Robles, M. M.; Pérez, O.; Orozco, M.; Williams, T. y Becerra, S. 2007. Fenología, eficiencia productiva y calidad de fruta de cultivares de naranjo en el trópico seco de México. Rev. Fitotec. Mex. 30(2):133-143.

Mora, G.; Robles, P.; López, J. I.; Flores, J.; Acevedo, G.; Domínguez, D. y González, R. 2014. Situación actual y perspectivas del manejo del HLB de los cítricos. Rev. Mex. Fitopatol. 32(2):108-119.

Ortega, L. D.; Ramírez, A. J.; Mendoza, E. E. y Villegas, A. 2013. Abundancia estacional de Diaphorina citri (Hemiptera: Liviidae) en plantaciones de cítricos en cazones, Veracruz, México. Acta Zoológica Mexicana. 29(2):317-333.

Paris, M. T.; Allan, S. A.; Hall, D. G.; Hentz, M. G.; Croxton, S. D.; Ainpudi, N. and Stansly, P. A. 2017. Effects of temperature, photoperiod, and rainfall on morphometric variation of Diaphorina citri (Hemiptera: Liviidae). Environ. Entomol. 46(1):143-158.

Pluke, R. W. H.; Qureshi, J. A. and Stansly, P. A. 2008. Citrus flushing patterns, Diaphorina citri (Hemiptera: psyllidae) populations and parasitism by Tamarixia radiata (Hymenoptera: Eulophidae) in Puerto Rico. Florida Entomologist. 91(1):36-42.

Qureshi, J. A. and Stansly, P. A. 2008. Rate, placement, and timing of aldicarb applications to control asian citrus psyllid, Diaphorina citri Kuwayama (Hemiptera: Psyllidae), in oranges. Pest Manag. Sci. 64(11):1159-1169.

Razeto, B. 2005. El limonero. Santiago de chile. Fundación para la innovación agraria. 21-36 pp.

Rencher, A. 2002. Methods of multivariate analysis. New York. Wiley-Interscience A John \& Sons, Inc. 727 p.

Robles, P. L. y Delgadillo, I. 2010. Protocolo de actuación para la detección del huanglongbing. Dirección de protección fitosanitaria. 39 p.

SIAP. 2019. Servicio de información agropecuaria y pesquera. SAGARPA. México.

Stansly, P. A. and Qureshi, J. A. 2007. Insecticidal control of Asian citrus psyllid through foliar applications on orange, 2006. Arthropod Management Tests. 29(1):14-23.

Tsai, J. H.; Wang, J. J. and Liu, Y. H. 2002. Seasonal abundance of the asian citrus psyllid, Diaphorina citri (Homoptera: Psyllidae) in southern florida. Florida Entomologist. 85(3):446-451. 